\title{
Multi dual-wavelength generation using InGaAsP/InP passive microring resonator with two sides apodized gratings
}

\begin{abstract}
Generation of dual-wavelength using passive semiconductor microring resonator with two sections of apodized grating with total length of $26 \mu \mathrm{m}$ on the right and left sides is presented here. In this research we use the time-domain travelling wave (TDTW) method to model and simulate the microring resonator made of InGaAsP/InP waveguide. Microring resonator based optical mirrors and band-limited reflectors have been the subject of intense investigations in recent years. Therefore, we propose the microring resonator made of InGaAsP/InP waveguide having two sides grating section. The grating sections have a trapezoidal profile. The gratings provide the means to suppress repetition of the reflection spectrum at every free spectral range (FSR) removed from the design wavelength. The total grating length is $26 \mu \mathrm{m}$ (each grating side has length of $13 \mu \mathrm{m}$ ), where the total circumference of the microring resonator is $526 \mu \mathrm{m}$. Propagation of the input Gaussian pulse (with $10 \mathrm{~mW}$ power and bandwidth of $0.76 \mathrm{ps}$ ) within the grating sections is presented, where it shows a good confinement of the propagation within the waveguide. As a result, multiple dual-wavelengths with tunable spacing within a range of $127 \mathrm{pm}(15.8 \mathrm{GHz})$ and $237 \mathrm{pm}$ (29.6 GHz) could be generated at the throughput port of the microring resonator. The dispersion of the grating section versus the total grating length and the frequency response of the throughput port output signals are presented. The generated dual-wavelength has many applications in optical sensing, radio frequency (RF) radiation, optical communication, optical switching, millimetre wave generators and biological research.
\end{abstract}

Keyword: Microring resonator; Apodized grating; InGaAsP/InP waveguide; Dualwavelength 